EDUCATIONAL

RESOURCE

Volume 12 lssue 42020

DOI: 10.21315/eimj2020.12.4.8

ARTICLE INFO

\title{
Discharge against Medical Advice and Transcending Boundaries in Ghanaian District Hospitals: Issues for Medical Learning
}

Accepted: 18-09-2020

Online: 30-12-2020

\author{
Kofi Tawiah Mensah
}

Agogo Presbyterian Hospital, Ghana, WEST-AFRICA

To cite this article: Mensah KT. Discharge against medical advice and transcending boundaries in Ghanaian district hospitals: issues for medical learning. Education in Medicine Journal. 2020;12(4):53-58. https://doi.org/10.21315/eimj2020.12.4.8

To link to this article: https://doi.org/10.21315/eimj2020.12.4.8

\section{ABSTRACT}

There is a case of a 47-year-old man who presented to the Agogo Presbyterian Hospital with a highgrade intestinal obstruction. Although the surgical expertise was available, limitations of laboratory and critical care services at the time necessitated a referral to a tertiary hospital. The patient and his relatives did not have the resources to pursue this treatment decision and opted for a discharge against medical advice (DAMA) to embark on faith-based treatment options. The attending clinician is sometimes frustrated in such complex scenarios by the inability to explore even the slightest survival chances that could result from life-saving surgery in the face of these resource limitations. This article focuses on the ethical and medicolegal dilemmas presented to clinicians by a patient's request for DAMA. Some pragmatic approaches have been proposed to guide the evaluation and resolution of such challenges at the level of Ghanaian district hospitals for the benefit of medical learning.

Keywords: Discharge, Ethical, Medicolegal, Patient, Surgery

Kofi Tawiah Mensah, Specialist Surgeon, Agogo Presbyterian Hospital, Box 27, Agogo, Ashanti-Akim North, Ghana, West-Africa | Email: tawiahmensahkofi@gmail.com

\section{INTRODUCTION}

Discharge against medical advice (DAMA) is often happens in Ghanaian clinical settings and medical trainees should be well informed of the dynamics and dilemmas involved in managing such situations well in advance. In the Ghana Health Service Patient's Charter, the client has the right to decide if they would like to be treated and what option of care is offered to them after receiving adequate information from the practitioner (1). However, there are situations where other factors influence some patients and their relatives to opt for DAMA, a decision that has been linked to less favourable outcomes (2). Some of these factors such as lack of financing for treatment (3), perceived lack of progress with hospital care and feelings of being misunderstood by caregivers (4). In a study by Malekzadeh et al. (5), some patients request for DAMA after perceived improvement in the medical condition, which they were admitted. From this latter finding, DAMA could result from a lack of quality and clarity in the communication between the clinician and patient (6). Medical trainees need to be aware of the ethical and medicolegal dilemmas that issues of an adult patient's request for DAMA and the resources at their disposal to address them while prioritising patient interests. 
Because interrogative studies on DAMA in Ghana are limited, this case report qualitatively examines the topical issues underpinning a decision for DAMA in an adult Ghanaian male admitted with a surgical emergency at the Agogo Presbyterian Hospital.

\section{CASE PRESENTATION}

A 47-year-old man presented to the Agogo Presbyterian Hospital with a high-grade intestinal obstruction from suspected postoperative intraperitoneal adhesions. He had an abdominal surgery two months earlier but the details of the operation were not available. On experiencing intermittent abdominal pains and constipation of increasing severity over the course of a month, that was also associated with progressive distension not responding to herbal remedies, he reported to a nearby health centre. From there, he was referred to the closest district hospital for treatment. After two days without improvement, he came to the secondary hospital and spent a further 20 hours before being referred.

He arrived very ill with clinical evidence of multiple physiological derangements including a markedly reduced urine output of $0.2 \mathrm{~mL} / \mathrm{kg} / \mathrm{h}$ that did not respond to fluid resuscitation. Abdominal X-rays confirmed a high-grade intestinal obstruction. The intention was to give him a chance by surgically intervening if there was the slightest improvement in the urine output with resuscitation.

The key issues in the management of this patient were threefold: Firstly, there was a severely impaired renal function, possibly, already with an established acute tubular necrosis. Secondly, the laboratory could not run serum electrolytes to allow for objective assessment and correction of derangements in preparation for surgery. Finally, his relatives could no longer come up with health care bill, and because he did not possess an active health insurance policy, they insisted on pursuing alternative treatment out of the hospital and produced a letter to that effect. At this point, his relatives were extensively informed about the options available and the need for a prompt referral to a tertiary hospital for continued care. The patient was counselled against the consideration of alternative medicine of a surgical abdomen. However, it was not immediately apparent how much this conversation had influenced any shift in their position on the subject of alternative medicine. The relatives managed to convince the patient, who also began to insist on being granted a discharge home.

Without regard to the DAMA letter, he was released from the secondary hospital with a referral, after about 20 hours stay, to a tertiary hospital where he could have received comprehensive laboratory workup, surgical intervention and critical care if he needed it. Alternative transportation had been organised by his relatives for this purpose. Subsequent feedback after this separation, however, confirmed that he was taken home for the intended alternative medicine but died a few hours after arrival.

\section{DISCUSSION}

Contrary to the scenario where majority of requests for DAMA in Western studies come from patients with some form of substance dependency or psychiatric disorder (7-9), this case report is a common finding in Ghana where the request stems from poor socioeconomic support (10) and belief systems. This finding resonates with other studies on DAMA carried out in the sub-region (3).

Faith-based healing (FBH) is currently well-advertised in Ghana with some providers claiming to possess remedies for conditions that doctors have failed to cure. The outcomes of alternative medicine in Ghana have not been objectively subjected to rigorous studies but are, generally, known to be poorer (11). The strong superstitions surrounding the practice of FBH limit the inquiry into its outcomes. This could be the 
result of client intimidation and gagging, particularly, when $\mathrm{FBH}$ results are adverse. For similar reasons, it is uncommon to find any legal action taken up against $\mathrm{FBH}$ service providers in Ghana.

It is notable that the patient employed some traditional remedies, initially, before reporting for the first time to a hospital with his condition. This is the story of most patients of low socioeconomic status living in rural and suburban Ghana. Many of such patients leaving hospitals with a DAMA return to the same $(7,12)$ or different hospitals after unsatisfactory stints with FBH. Some of these returning clients, by that time, would have missed time-sensitive "golden" opportunities to obtain more acceptable and less expensive treatment. As an example, one study reported that $75 \%$ of clients seeking the services of traditional bone-setters in northern Ghana, had initially sought DAMA from the hospital (11).

When confronted with the emotionally stressful crossroads of a DAMA request from an adult patient, clinicians would benefit from existing decision-making guidelines $(10,13)$ but these are often unavailable to the Ghanaian doctor. More than half of Ghanaian patients do not know their rights enshrined in the patient charter (14); this is an indication of the limited scope of health literacy in the nation. Wellenlightened patients and their relatives can be expected to rely less on superstition and be more agreeable with doctors who try to negotiate with them on safer treatment options other than a DAMA.

Since the index patient had no money of his own to spend on his health and had to rely on his indigent household, the family assumed a major decision-making role in the patient's care. Not having an active subscription to the National Health Insurance Scheme (NHIS), a common finding among persons of lowsocioeconomic status, was a massive disadvantage for this client who had to resort to the "cash and carry" process.
DAMA has been found to be strongly associated with patients having complex social issues (8). In many low-income settings, patients tend to consider themselves as fortunate recipients of whatever limited medical expertise is available to them or as much as they can afford - whichever one comes first. A year ago in Ghana, when a critically ill person needed a referral, the patient's sponsors were required to make upfront payment for ambulance services as soon as a vehicle was available. The cost of out-of-pocket medical care in Ghanaian tertiary hospitals is rather high for which reason uninsured, low social class patients hesitate to embark on tertiary referrals, sometimes even when they could lose their lives without it.

It is unethical, professionally, to carry out a procedure whose potential risks could outweigh the benefits to the patient (1). Notwithstanding this, a few questions may arise in such complex scenarios. Where the physician, institutional setup, patient and their sponsors are at their wits end, should a DAMA request, of all alternatives, be acceptable? Is an endorsed DAMA request on a regular sheet authoritative enough to be granted? Can the clinician withhold a DAMA request even when the hospital can no longer render additional treatment except a referral - one which a patient's sponsors cannot afford or are unwilling to pursue? Can the clinician, institution or law court be empowered to compel a patient's sponsor to effect a prescribed tertiary referral, failure of which could result in legal action? The stark reality is that there are no prescribed guidelines to help the practitioner navigate the stormy course of a DAMA request in many Ghanaian hospitals.

On the other hand, there is a chance to successfully perform a life-saving laparotomy for such a patient described above under the supervision and expertise of a specialist surgeon in the district hospital amidst the complexities surrounding the diagnosis. Some investigations such as the electrolyte assay and renal function tests have become 
available in Ghanaian district hospitals only over the last decade. Prior to this period, the haemoglobin level and urine output measurements were all that were available before going to surgery. This practice, certainly, had its drawback for some patients in whom occult biochemical derangements were missed. Nonetheless, an over-insistence on superfluous tests as prerequisites for "safe surgery" in the primary health care setting, could increasingly restrain surgeons, ultimately depriving some indigent patients of a chance to live. When the surgical expertise is available, it is possible to make the most out of the resources at hand.

Without disregarding the principle of non-maleficence, where ideal clinical circumstances do not exist, patients probably have the right to take their chances, following a voluntary informed consent that captures their acceptance of the associated risks. Clinical practice in low-resource settings sometimes bulldozes its way through challenging uncertainties. Doctors should not give up too soon on such deteriorated cases. This proposition draws similarities with experimental drug or surgical therapy for terminal conditions where there are really no guarantees on outcomes. Critically ill surgical patients, similarly perhaps, would like to see their doctors become more confident and creative in stepping out of some of their professional comfort zones to save patient lives.

The legal basis for this proposition, however, needs to be further developed and clarified. In the hospital, when there is nothing new, affordable or effective enough to offer critically ill patients, it should, probably, not be too surprising when patients present a DAMA request. Patients sometimes seem to be telling doctors that, "what is sure to happen here while doctors do nothing, can also happen in the comfort of our homes". As opposed to being granted a DAMA to resort to $\mathrm{FBH}$, it could be better for critically ill surgical patients to "have a go" at surgery, however the great risk sometimes, within the controlled environments of the hospital. Perhaps, it is a time for clinicians listened more attentively.

To this end, it is relevant that medical students and interns receive the needed exposure to the complex issues of a DAMA request and provided with frameworks for decision-making. There would be the need to acquire the capacity to anticipate a DAMA request based on some circumstantial indicators and adopt a preventive approach to addressing the matter. However, the reality to be faced by the district doctor in Ghana is usually that of being left with little choice, but to grant a DAMA even when the requesting document is not simply and convincing enough because the relatives sometimes threaten to abandon the patient in the hospital if the DAMA does not proceed.

An inclusive stakeholder approach involving doctors, faith healers and non-governmental organisations would allow more openness in the patient treatment and referral system. Faith healers may not be stamped out anytime soon but until then, they could be trained to be capable of directing such seriously ill patients to the hospitals as the case should rightly be. As yet, no clinician in Ghana has faced any legal action because of granting or denying a request for DAMA. The story, however, might not remain the same in the coming years, and hence, some of the above pragmatic alternatives to a DAMA request need to be devised for the future. A request for DAMA should no longer remain a medicolegal 'gray area' in Ghanaian clinical practice.

\section{CONCLUSION}

DAMA will probably be an inherent part of clinical care in Ghana for which trainees would benefit from adequate exposure and preparation to deal with its multifaceted influences and consequences. Trainee clinicians must be equipped with the negotiation skills to weaken the factors influencing patients to pursue a DAMA. Stronger and comprehensive legislative 
framework should be developed to empower and protect institutions and clinicians in their quest to safeguard patient health especially when confronted with the conflicting interests of sponsoring relatives. Although it may not be appropriate in all situations, critically ill patients who provide voluntary informed consent for specialistled life-saving surgery at the district hospital, despite the lack of full laboratory work-up, should, perhaps, be offered that chance rather than be granted a DAMA. Since poverty is a common determinant for a DAMA request, creative ways are needed to correct the shortfall in the NHIS coverage among indigents.

\section{REFERENCES}

1. Ghana Health Service. The patient's charter. 2017 [cited 2020 June 6]. Available from: https://ghanahealthservice.org/ghssubcategory.php?cid $=2 \&$ scid $=46$

2. Muftau JB, Anthonia OC, Chinwe I, Oluwafemi A, Ganiyu A, Haroun A, Chinwe E, Joshua A. Prospective evaluation of cases of discharge against medical advice in Abuja, Nigeria. Scientific World Journal. 2015:314817. https://doi. org/10.1155/2015/314817

3. Ibekwe RC, Muoneke VU, NnebeAgumadu UH, Amadife MA. Factors influencing discharge against medical advice among paediatric patients in Abakaliki, Southeastern Nigeria. J Trop Pediatr. 2009;55(1):39-41. https://doi.org/10.1093/ tropej/fmn 100

4. Brzezinski M, Gregersen M, Schuch LG, Sawatzki R, Chen JW, Gauger G, Kukreja J, Cason B. Discharge against medical advice in surgical patients with posttraumatic stress disorder: a case report series illustrating unique challenges. Case Reports in Anesthesiology. 2017:3045907. https://doi. org/10.1155/2017/3045907
5. Malekzadeh R, Araghian Mojarad F, Amirkhanlou A, Sarafraz S, Salmanpour M. Causes of discharge against medical advice in hospitals affiliated with Mazandaran university of medical sciences, 2014. Journal of Mazandaran University of Medical Sciences. 2016;26(140):95-102. https://doi. org $/ 10.5455 / \mathrm{msm} .2014 .26 .203-207$

6. Franks $\mathrm{P}$, Meldrum $\mathrm{S}$, Fiscella $\mathrm{K}$. Discharges against medical advice: are race/ ethnicity predictors? J Gen Intern Med. 2006;21(9):955-60. https://doi.org/10.1111/ j.1525-1497.2006.00505.x

7. Anis AH, Sun H, Guh DP, Palepu A, Schechter MT, O'Shaughnessy MV. Leaving hospital against medical advice among HIVpositive patients. CMAJ. 2002;167(6):6337.

8. Williams $\mathrm{T}$, Woolley $\mathrm{M}$, Al-Omary $\mathrm{M}$. Discharge against medical advice: a tertiary referral centre experience. Heart Lung and Circulation. 2019;28(Supp.4):S343. https:// doi.org/10.1016/j.hlc.2019.06.485

9. Duñó R, Pousa E, Sans J, Tolosa C, Ruiz A. Discharge against medical advice at a general hospital in Catalonia. General Hospital Psychiatry. 2003;25(1):46-50. https://doi. org/10.1016/S0163-8343(02)00253-0

10. Berger JT. Discharge against medical advice: ethical considerations and professional obligations. J Hosp Med. 2008;3(5):403-8. https://doi.org/10.1002/jhm.362

11. Kuubiere CB, Abass A, Majeed SF. Patients preference for traditional bonesetters in Northern Ghana. Religion. 2015;2:2-5.

12. Ashrafi E, Nobakht S, Keykaleh MS, Kakemam E, Hasanpoor E, Sokhanvar M. Discharge against medical advice (DAMA): causes and predictors. Electron Physician. 2017;9(6):4563-70. https://doi. org/10.19082/4563 
13. Chin J, McDougall R. Beyond the waiver: an ethical approach to discharge against medical advice. Camb Q Healthc Ethics. 2018;27(2):348-52. https://doi.org/10.1017/ S0963180117000676.
14. Abekah-Nkrumah G, Manu A, Atinga RA. Assessing the implementation of Ghana's patient charter. Health Education. 2010:110(3). https://doi. org/10.1108/09654281011038840 